\title{
Workflow Management in Electronic Commerce
}

\author{
Paul Grefen \\ Computer Science Department, University of Twente \\ P.O. Box 217, 7500 AE Enschede, Netherlands \\ www. cs. utwente.nl/ grefen \\ grefen@cs.utwente.nl
}

\section{Introduction}

In electronic commerce scenarios, effectiveness and efficiency of business process execution are of paramount importance for business success. Even more than in traditional commerce scenarios, they determine the chances of survival of organizations in fast moving, highly competitive electronic markets. To obtain the required levels of effectiveness and efficiency, well-structured automated business process support is required. For typical business-to-consumer (B2C) electronic commerce, process support is usually of an intra-organizational nature. For businessto-business (B2B) electronic commerce, however, process support across organizational boundaries is often required as the basis for virtual enterprises.

This tutorial addresses the application of workflow management (WFM) for process support in both these cases. The tutorial is organized into three parts. In the first part, we pay attention to 'classical' workflow management in the context of a single organization. In the second part, we extend this to workflow management across the boundaries of organizations. In the third part, we further extend this model by making service processes - implemented as workflows - the objects traded in ecommerce scenarios. We outline each part in the sections below.

\section{Intra-organizational Workflow Management}

The basics of intra-organizational workflow management are treated in short in the first part of this tutorial and placed in the context of electronic commerce. We start with discussing the overall goal and main functionalities of workflow management. Then, we pay attention to both conceptual modeling and high-level architectural aspects.

The main workflow management concepts are discussed and placed in a general workflow management model. This model consists of process, organization and information submodels. The right way of coupling these submodels is essential for flexible workflow management.

The conceptual workflow model is a basis for workflow management systems that manipulate instances of these models. The overall architecture of WFM systems is treated by means of reference architectures. We show how WFM systems fit in the context of information infrastructures. 


\section{Cross-Organizational Workflow Management}

Cross-organizational workflow management (XO-WFM) is required in B2B ecommerce scenarios to support business processes spanning two (or more) organizations. XO-WFM requires specific extensions of basic workflow support as discussed in Part 1 of this tutorial, both conceptually and architecturally.

These extensions are treated in this part of the tutorial. We discuss which additional requirements have to be met by XO-WFM with respect to intraorganizational workflow management. This is the basis for XO-WFM concepts and models on the one hand and XO-WFM architectures on the other hand. We show how XML can be used to achieve interoperability in workflow specification between organizations. We discuss how chosen organization forms for e-commerce influence the choice of abstract architectures for workflow management.

$\mathrm{XO}-\mathrm{WFM}$ is treated in the context of a number of B2B e-commerce application areas. One specific area - in which workflow management is the central ingredient is further elaborated in the next part of this tutorial.

\section{Workflow Management in Dynamic Service Outsourcing}

An important class of B2B electronic commerce is dynamic service outsourcing, in which services are the objects of commerce. In this paradigm, consumer organizations buy the execution of services by provider organizations. Fine-grained monitoring and control of outsourced services is important in scenarios where complex, missioncritical processes are outsourced. Dynamic service outsourcing requires models and mechanisms for service trading between organizations and service enactment across organization boundaries - hence a tight coupling of e-commerce and workflow management must be supported.

The third part of the tutorial goes into the role of workflow management for service enactment in this context. We explain the concept of service outsourcing and illustrate the paradigm by means of an abstracted architecture. We show that the concept of electronic contract is required to formalize the business relationship between partners engaging in outsourcing. Workflow specifications of the services to be outsourced are part of these contracts. We discuss a high-level conceptual model for electronic contracts and show how this can be represented in an XML-based contract specification language. We end the tutorial by briefly presenting two business cases in which the service outsourcing paradigm is applied. 